\title{
4D cardiac magnetic resonance imaging, 4D and 2D transthoracic echocardiography: a comparison of in-vivo assessment of ventricular function in rats
}

Aus der Abteilung für Kinderkardiologie des Universitätsklinikums

Erlangen

Leiter: Prof. Dr. med. S. Dittrich

\author{
der Medizinischen Fakultät \\ der Friedrich-Alexander-Universität \\ Erlangen-Nürnberg \\ zur \\ Erlangung des Doktorgrades Dr. med.
}

vorgelegt von

Hedwig Christine Stegmann 


\begin{abstract}
Als Dissertation genehmigt von der
Medizinischen Fakultät der Friedrich-Alexander-Universität

Erlangen-Nürnberg
\end{abstract}

Vorsitzender des Promotionsorgans: Prof. Dr. med. Markus Neurath

Gutachter:

Prof. Dr. Sven Dittrich

Gutachter:

Prof. Dr. Tobias Bäuerle

Tag der mündlichen Prüfung:

23. März 2021 


\section{Inhaltsverzeichnis}

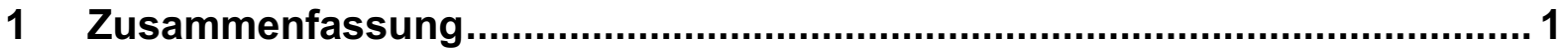

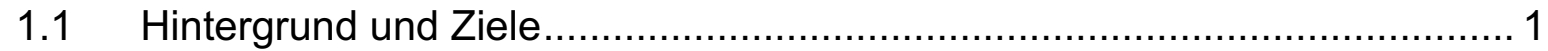

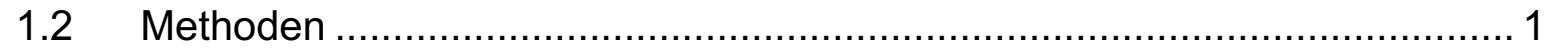

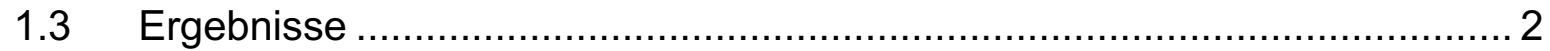

1.4 Praktische Schlussfolgerungen und Ausblick ..................................... 2

2 Einleitung und Einordnung in den fachwissenschaftlichen Kontext............ 3

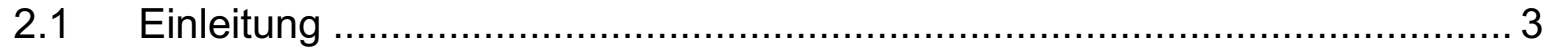

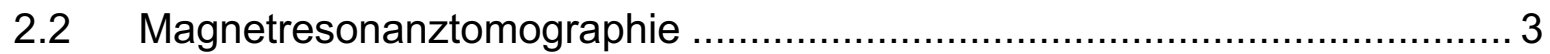

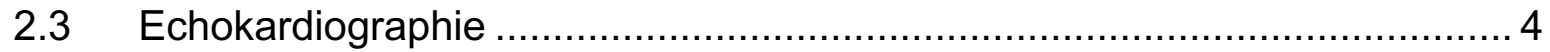

2.4 Rattenmodelle in der präklinischen kardiovaskulären Forschung ................ 7

3 Forschungsstand der kardialen Bildgebung bei Nagetieren ........................ 8

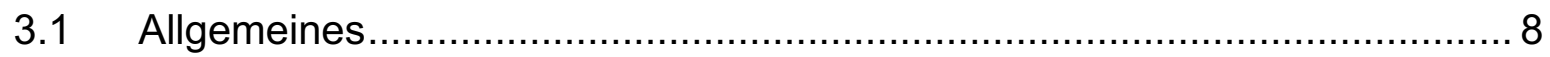

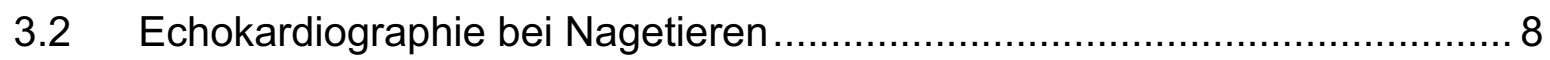

3.3 Kardiale Magnetresonanztomographie bei Nagetieren ........................... 13

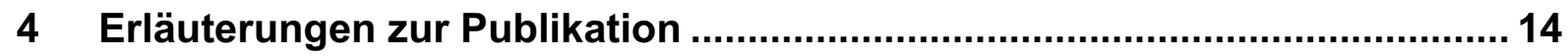

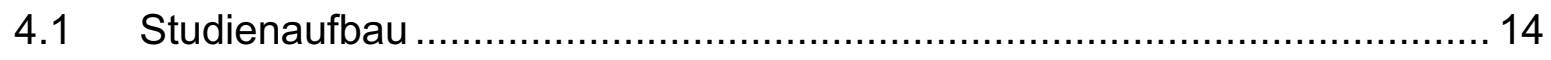

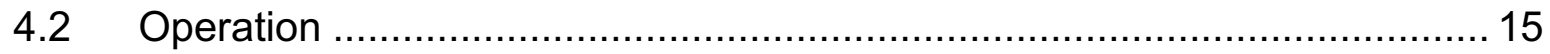

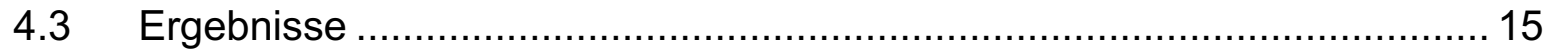

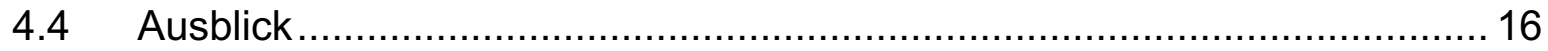

5 Originalpublikation: „4D cardiac magnetic resonance imaging, 4D and 2D transthoracic echocardiography: a comparison of in-vivo assessment of ventricular function in rats“ ..........................................................................17

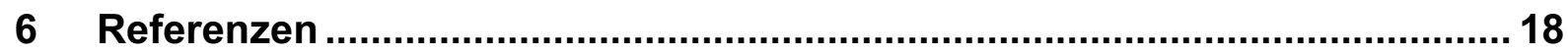

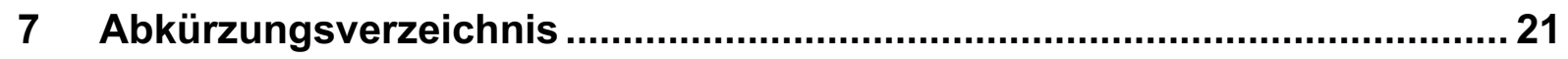

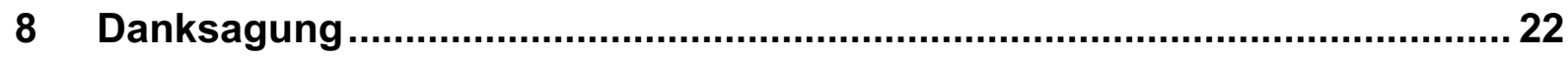




\section{Zusammenfassung}

4D kardiale Magnetresonanztomographie, 4D und 2D transthorakale Echokardiographie: Ein Vergleich von in-vivo Untersuchungen der Herzfunktion an Ratten

\subsection{Hintergrund und Ziele}

Die Herz-Kreislauf-Forschung ist die Grundlage unseres Verständnisses der Herzfunktion und Herz-Kreislauf-Erkrankungen. Zuverlässige kardiale Bildgebungsverfahren sind hierbei unerlässlich zur Gewinnung valider Ergebnisse. Zur Untersuchung der Herzfunktion stehen unterschiedliche bildgebende Verfahren zur Verfügung, die, je nach Fragestellung, ihre Vor- und Nachteile haben. In der präklinischen forschung gilt die vierdimensionale kardiale Magnetresonanztomographie (4D CMR) als Goldstandard für die In-vivo-Bewertung der ventrikulären Funktion bei Nagetieren. Durch die technischen Fortschritte der letzten Jahre ist es nun möglich, mithilfe der Echokardiographie das gesamte Herz abzubilden. Durch diese Weiterentwicklung stellt vierdimensionale Echokardiographie (4DE) eine mögliche alternative Bildgebung zur 4D CMR dar.

Aufgrund fehlender Forschungsarbeiten zu diesem Thema war es Ziel der vorliegenden Studie, die Genauigkeit und Reproduzierbarkeit von 4DE im Vergleich zur 4D CMR an Ratten zu untersuchen.

\subsection{Methoden}

Es wurden 26 junge Sprague-Dawley-Ratten untersucht. 20 dieser Ratten wurden sowohl echokardiographischen Aufnahmen (2D und 4D) als auch 4D CMR unterzogen. 4D CMR wurde mit einem 7 T-Scanner, 2D- und 4D-Echokardiographie mit einem $40 \mathrm{MHz}$-Linearschallkopf durchgeführt. Beide Geräte wurden speziell für Kleintiere entwickelt.

Als Kontrollgruppe wurden fünf der 26 Ratten zwei Wochen vor den Scans einer operativen Ligatur der oberen und unteren Hohlvene unterzogen, um den kardialen Zufluss zu reduzieren. 
Um die Reproduzierbarkeit der Echokardiographie beurteilen zu können, wurden sechs weitere Ratten im Alter von vier Wochen durch zwei verschiedene Personen jeweils drei echokardiographischen Untersuchungen unterzogen.

\subsection{Ergebnisse}

Untersucht wurden die Korrelationen zwischen 4D CMR, 4DE und 2DE für die linksventrikuläre Ejektionsfraktion (LVEF) und die Reproduzierbarkeit von 4DE und 2DE. Es wurde eine hochsignifikante Korrelation zwischen 4DE und 4D CMR beobachtet $(r=0,57 ; p<0,01)$. Daraus folgt, dass die 4DE Untersuchungsergebnisse liefert, die genauso präzise wie die der 4D CMR sind. Sowohl die Untersuchungszeit und die damit verbundene Sedierungsdauer als auch der technische Aufwand sind bei 4DE geringer.

Die 4DE-Ergebnisse zeigen eine sehr geringe Intraobserver- und InterobserverVariabilität und sind daher gut reproduzierbar.

\subsection{Praktische Schlussfolgerungen und Ausblick}

Die Ergebnisse zeigen, dass die 4DE eine verlässliche Alternative zur 4D CMR ist. Die kürzere Sedierungsdauer der Tiere und die bessere, dezentrale Verfügbarkeit sind vor allem bei longitudinalen Studien von Vorteil. Für die präklinische Forschung ist die 4DE daher ein großer Fortschritt. 


\section{Einleitung und Einordnung in den fachwissenschaftlichen}

Kontext

\subsection{Einleitung}

Die Möglichkeiten und die Genauigkeit der Ergebnisse der bildgebenden Verfahren werden durch technische Weiterentwicklungen kontinuierlich verbessert. Deshalb ist es unerlässlich, auf dem aktuellen Stand der Forschung und Technik zu bleiben. Je nach Fragestellung stehen verschiedene Verfahren zur Verfügung, die auf unterschiedlichen physikalischen Grundlagen wie Magnetresonanz, Ultraschall oder Röntgenstrahlung basieren. Je nachdem ob die Verfahren auf Magnetresonanz, Ultraschall oder Röntgenstrahlung aufbauen, bieten diese Vor- und Nachteile. Des Weiteren unterscheidet man zwischen statischen und dynamischen Darstellungen. Aus diesem Grund ist es entscheidend, das für die jeweilige Fragestellung optimale Verfahren auszuwählen. In der folgenden Studie wird die kardiale MRT mit der 2D und 4D-Echokardiographie an Ratten verglichen. Deshalb wird im Folgenden auf diese drei Verfahren näher eingegangen.

\subsection{Magnetresonanztomographie}

Die Magnetresonanztomographie oder auch Kernspintomographie gehört zur Untergruppe der Schnittbildverfahren und arbeitet mit sehr starken, konstanten Magnetfeldern zwischen 3 und 9,4 Tesla und Radiowellen. Dieses bildgebende Verfahren wurde erstmals in den 1970er Jahren eingesetzt und ist aus der modernen Radiologie nicht mehr wegzudenken [1, 2]. Die Magnetresonanztomographie beruht auf der physikalischen Eigenschaft des Kern-Spins. Der Spin ist der Eigendrehimpuls von Atomkernen mit ungerader Protonen- oder Neutronenzahl. Das Proton im Wasserstoffatom dreht sich hierbei um sich selbst und ist dadurch magnetisch. Im Normalzustand sind die Spins nicht geordnet. Sobald jedoch ein starkes externes Magnetfeld anlegt wird, richten sich die Protonen parallel oder antiparallel zur Feldrichtung aus. Während der Messung gibt der Magnetresonanztomograph senkrecht zur Ausrichtung des Magnetfelds hochfrequente Radiowellen auf den Körper ab. Hierdurch wird die Ausrichtung der Wasserstoffkerne im Magnetfeld verändert (Transversalmagnetisierung). Im Anschluss an jeden Radiowellen-Impuls 
richten sich die Wasserstoffkerne wieder in Längsrichtung des Magnetfeldes aus. Diese Änderungen der Magnetisierung werden durch eine Messspule registriert und so in elektrische Impulse umgewandelt, welche gemessen und $\mathrm{zu}$ Bildern zusammengesetzt werden können [3].

Da das Wasserstoffatom am stärksten auf das Magnetfeld reagiert, liefert die MRT vor allem bei stark wasserhaltigem Gewebe eine sehr genaue Darstellung und eignet sich somit für die Darstellung von Weichteilen. Dies resultiert aus den unterschiedlichen Fett- und Wassergehalten der verschiedenen Gewebearten [4]. Die Differenzierung verschiedener Gewebearten kann durch den Einsatz spezieller Kontrastmittel zusätzlich verbessert werden. Die kardiovaskuläre Magnetresonanztomographie erlaubt neben der Darstellung des Myokards durch spezielle Sequenzen eine Gewebecharakterisierung, den Nachweis von Durchblutungsstörungen sowie die Darstellung des Blutflusses in den Gefäßen $[5,6]$. Vorteil des MRTs ist vor allem die hohe Bildqualität, die jedoch mit einer langen Untersuchungsdauer einhergeht. Außerdem werden für dieses Verfahren keine Röntgenstrahlen benötigt, es kommt also zu keinerlei Strahlenexposition. Allerdings stellen Metallimplantate verschiedener Art wie zum Beispiel Herzschrittmacher und Cochlea-Implantate zum Teil Kontraindikationen dar [1, 7, 8]. Außerdem kann eine starke Klaustrophobie des Patienten die MRT-Untersuchung erschweren oder unmöglich machen. Bei pädiatrischen Patienten ist für eine MRT häufig eine Sedierung notwendig [9]. Im Vergleich zur Sonographie ist die Kernspintomographie ein teures Verfahren. Bei Nagetieren und damit vor allem in der präklinischen Forschung fällt hauptsächlich die lange Untersuchungsdauer und die damit für das Tier verbundenen längere Sedierungszeit ins Gewicht. Die lange Untersuchungsdauer macht longitudinale Studien mit mehrfachen MRT-Kontrollen schwierig umsetzbar.

\subsection{Echokardiographie}

Unter Echokardiographie versteht man die sonographische Untersuchung des Herzens. Sonographie ist ein bildgebendes Verfahren, bei dem das Aussenden und Empfangen von Ultraschallwellen zur Untersuchung von Strukturen und Gewebe genutzt wird. Es handelt sich um eine nicht-invasive, schmerzlose und strahlenexpositionsfreie Anwendung mit hoher Verfügbarkeit. In der Diagnostik 
verwendet man üblicherweise Frequenzen zwischen $1 \mathrm{MHz}$ und $40 \mathrm{MHz}$ [10]. Das Ultraschallgerät enthält Elektronik für die Schallerzeugung, Signalverarbeitung und Signaldarstellung. Im Kopf der Sonde sind kleine Piezokristalle, sogenannte Transducer, angeordnet. Über eine angelegte Wechselspannung verschieben sich periodisch die Ladungsschwerpunkte in den Piezokristallen. Durch diese oszillierende Bewegung erzeugt der Transducer Schallwellen, die in Form von Ultraschallwellen den Schallkopf verlassen. Diesen Effekt bezeichnet man als den inversen piezoelektrischen Effekt. Die ausgesendeten Schallwellen werden vom betrachteten Gewebe unterschiedlich stark gestreut und reflektiert. Die Reflexionsintensität ist für jedes Medium charakteristisch und wird auch als Wellendwiderstand oder akustische Impedanz bezeichnet. Die Ultraschallwellen, die auf Grenzflächen zweier Gewebe unterschiedlicher akustische Impedanz treffen, werden teilweise reflektiert, während die übrige Schallenergie die Grenzfläche passiert. Das Ausmaß der Schallreflexion ist umso größer, je mehr sich die beiden Impedanzen unterscheiden [11]. Am Übergang zwischen Gewebe und Luft erfolgt deshalb eine fast vollständige Reflexion. Aus diesem Grund benötigt man Ultraschallgel, um eine luftblasenfreie Kontaktfläche zwischen Schallkopf und Körperoberfläche zu ermöglichen [12, 13]. Die reflektierten Schallwellen werden im Schallkopf mithilfe des direkten piezoelektrischen Effektes detektiert. Hierbei üben die Schallwellen eine mechanische Kraft auf die Piezokristalle aus, die hierdurch geringfügig verformt werden. Dadurch verschieben sich die Ladungsschwerpunkte der Kristalle gegeneinander, wodurch sich eine elektrische Spannung abgreifen lässt. Diese wird dann hinsichtlich Frequenz, Laufzeit und Amplitude ausgewertet und über verschiedene Graustufen quantifiziert. Im Herzen reflektiert das Myokard die Ultraschallwellen stärker (hyperechogen) als das Blut (hypoechogen). Strukturen mit hoher Echogenität werden hell oder sogar weiß und Strukturen mit geringer Echogenität dunkel dargestellt [14].

Für die sonographische Untersuchung des Herzens gibt es zwei Möglichkeiten: Die transthorakale Echokardiographie (TTE) und die transösophageale Echokardiographie (TEE). Die TTE ist ein einfaches und schnelles Verfahren zur Darstellung des Herzens und zur Beurteilung der Herz- sowie der Klappenfunktion. Es wird sowohl in der Klinik als auch in der präklinischen Forschung flächendeckend eingesetzt. Da der Schallkopf vom Untersucher geführt wird, ist dieses Verfahren im Vergleich zur MRT stark untersucherabhängig [15, 16]. 
Für die TEE wird eine Ultraschallsonde über den Pharyngealraum in den Ösophagus eingeführt. Deshalb wird für die Dauer der Untersuchung eine Sedierung benötigt, wodurch die Untersuchung aufwändiger ist. Vorteil bei diesem Verfahren ist jedoch, dass der Schallkopf im Ösophagus dem Herzen näher ist. Hierdurch wird eine verbesserte sonographische Darstellung und dementsprechend auch eine höhere Aussagekraft erreicht. Die TEE wird vor allem zur Untersuchung der Herzklappen und der Vorhöfe eingesetzt $[17,18]$.

Die Qualität der sonographischen Bilder ist physikalisch limitiert. Um eine hohe Auflösung des resultierenden Bildes zu bekommen gilt: Je höher die Frequenz, desto besser die Auflösung. Allerdings nimmt die Eindringtiefe der Ultraschallwellen mit steigender Frequenz ab [19]. Man spricht hier von frequenzabhängiger Dämpfung. Deshalb muss die Frequenz in Abhängigkeit von der Eindringtiefe gewählt werden. Hochfrequenz-Schallköpfe, welche mit Frequenzen zwischen 30 und $70 \mathrm{MHz}$ arbeiten, haben lediglich eine Eindringtiefe von ungefähr $10 \mathrm{~mm}$. Folglich ist eine Anwendung in der Erwachsenenkardiologie ausgeschlossen. Für die Echokardiographie an Kleintieren ist diese frequenzabhängige Dämpfung ein deutlich geringeres Problem, da bei diesen, im Vergleich zum Menschen, geringe Eindringtiefen von ungefähr $10 \mathrm{~mm}$ ausreichen. Der Bereich von 2 bis $5 \mathrm{MHz}$ stellt einen guten Kompromiss für die Anwendung am Menschen dar, da in diesem Frequenzbereich die Absorption wenig ins Gewicht fällt.

Ein weiterer wichtiger Faktor in der Echtzeit-Bildgebung ist die zeitliche Auflösung. Diese wird bestimmt durch die Bildwiederholrate, also die Anzahl der unbewegten Bilder, die pro Sekunde visualisiert werden. Die Bildwiederholrate ist abhängig von vielen Faktoren, unter anderem der Eindringtiefe, der Anzahl der Abtastlinien und der Impulsfrequenz, also die Anzahl der gesendeten Impulse in einer definierten Zeit. Die großen Vorteile der Echokardiographie sind die schnelle Verfügbarkeit und einfache Anwendung bei ausreichender Erfahrung des behandelnden Arztes. Des Weiteren kann sie direkt am Patientenbett durchgeführt werden und ist dadurch sowohl in der notfallmäßigen Versorgung als auch bei Routinekontrollen das Standardverfahren kardialer Bildgebung [20]. 


\subsection{Rattenmodelle in der präklinischen kardiovaskulären Forschung}

Tiermodelle sind seit vielen Jahren wesentlicher Bestandteil der präklinischen HerzKreislauf-Forschung und sowohl für das pathologische Verständnis als auch für neue therapeutische Ansätze relevant. Die Auswahl des Versuchstiers sollte stets sehr sorgfältig erfolgen, da es die Studienergebnisse und vor allem auch die Übertragbarkeit der Ergebnisse auf den Menschen stark beeinflusst. Hier haben sich über die letzten Jahrzehnte Nagetiere als Tiermodelle in der Herz-Kreislauf-Forschung bewährt [21, 22]. Insbesondere Ratten und Mäuse werden häufig eingesetzt [23]. Physiologisch und anatomisch sind das Ratten- und das Mäuseherz dem Menschenherzen sehr ähnlich. So sind vor allem die Kontraktilität und die damit einhergehende Auswurffraktion zwischen Menschen und Ratte sehr gut vergleichbar. Hinzu kommt, dass inzwischen das Erbgut von Mäusen und Ratten vollständig entschlüsselt ist [24], und zu 90\% mit dem des Menschen übereinstimmt [25]. Dies macht es möglich, genetisch modifizierte Tiere mit pathologischen Phänotypen wie zum Beispiel einem Ventrikelseptumdefekt oder einer arteriellen Hypertonie in großer Anzahl mit relativ geringem Aufwand zu züchten [26]. Gegenüber größeren Tierarten haben die kleinen Nagetiere außerdem den Vorteil, dass aufgrund der kurzen Generationszeit mit einer Wurfgröße von fünf bis 20 Jungtieren homogene Kohorten in benötigter Anzahl zeitnah zur Verfügung stehen [27]. Im Gegensatz zur Maus erleichtert die größere Körpergröße der Ratte eine Vielzahl von Untersuchungen, deshalb wurden die Untersuchungen an Ratten durchgeführt. 


\section{Forschungsstand der kardialen Bildgebung bei Nagetieren}

\subsection{Allgemeines}

Die Zuverlässigkeit und Genauigkeit kardialer Bildgebungsmethoden machen einen elementaren Teil der Herzkreislaufforschung aus. Die Entwicklung von Soft- und Hardware schreitet stetig voran. Es ist unerlässlich auf dem aktuellen Forschungsstand zu bleiben und die technischen Entwicklungen soweit möglich in die eigene Forschung zu integrieren.

In der präklinischen Herzkreislaufforschung werden überwiegend Nagetiere genutzt. Allerdings ist die Bildgebung an Nagetieren durch andere Faktoren limitiert als beim Menschen. Hier ist vor allem die hohe Herzfrequenz von 300 bis 400 Schlägen die Minute und die hohe Atemfrequenz von 30 bis 40 Atemzüge die Minute zu nennen. Erschwerend kommt die geringe Körpergröße der Tiere hinzu, die für exakte Messungen eine höhere räumliche Auflösung der Bildgebung erforderlich macht. In den folgenden beiden Unterkapiteln wird auf den Stand der Forschung für Echokardiographie und Magnetresonanztomographie bei Nagetieren getrennt eingegangen. Die anschließend eingefügte Originalpublikation stellt eine Verbindung zwischen den Methoden her.

\subsection{Echokardiographie bei Nagetieren}

Wie bereits beschrieben, sind die technischen Anforderungen an Kleintierultraschallgeräte nicht die gleichen, die an Ultraschallgeräte für Menschen gestellt werden. Durch diese physiologischen Voraussetzungen werden kleinere Schallköpfe mit höheren Frequenzen benötigt. Des Weiteren müssen die Ratten für die Dauer der Untersuchung sediert werden, damit man die Tiere in Rückenlage echokardiographisch untersuchen kann. Daher hat sich die Echokardiographie an Kleintieren erst im letzten Jahrzehnt zunehmend etabliert, während die Echokardiographie am Menschen schon lange zu den Standarduntersuchungen mit hoher Aussagekraft gehört [28].

Für den transthorakalen Ultraschall an Nagetieren nutzt man überwiegend die folgenden Formate: B-Mode (brightness mode), M-Mode (motion mode), DopplerSonographie und 3D- beziehungsweise 4D-Ultraschall. Seit 2016 steht bei einigen 
Geräten außerdem die EKG-synchronisierte Kilohertz-Visualisierung (EKV) zur Verfügung, wodurch die Bildklarheit deutlich erhöht werden konnte.

Die bisher verfügbaren Analysen aus den Standardmodi wie dem B-Mode und dem MMode basieren auf geometrischen Modellen wie Ellipsen oder Kugeln, um die Ventrikelvolumina zu ermitteln. Auch wenn diese geometrischen Annäherungen sowohl in der präklinischen als auch in der klinischen Bildgebung eine breite Anwendung finden, sinkt die Aussagekraft bei abweichender Ventrikelform und eingeschränkter Herzfunktion, wie es bei kardiologischen Erkrankungen häufig der Fall ist $[29,30]$. Die Notwendigkeit von 3D-Messungen wurde in vorherigen Publikationen als Weiterentwicklung gefordert, allerdings waren die technischen Grundlagen hierfür noch nicht gelegt $[19,31]$.

B-Mode: Der B-Mode produziert ein zweidimensionales Schwarz-Weiß-Bild in Echtzeit. In dieser Darstellung lassen sich Aussagen über Kammergröße und Herzfunktion treffen, ohne jedoch genaue quantitative Werte zu liefern. Ein Herzzyklus kann als Bildschleife, ein sogenannter Cineloop, mehrfach hintereinander und in langsamer Geschwindigkeit abgespielt werden. In Cineloop-Bildern kann das Endokard des linken Ventrikels getraced werden und damit die kardiale Auswurffraktion berechnet werden. Hierfür legt der Untersucher eine Markierung auf das Endokard, welche dann bei Kontraktion des Ventrikels automatisch auf dem Endokard bleibt, der Kontraktion des Myokards folgend. Typische Schnittachsen sind hier die parasternale lange Achse (PSLAX) und die parasternale kurze Achse (PSAX). In diesen jeweiligen Einstellungen wird dann ein einzelner Schnitt durch das Herz abgebildet. Der rechte Ventrikel ist im B-Mode schlecht darstellbar, da häufig das Sternum Teile des rechten Ventrikels verdeckt und eine vollständige Einsicht unmöglich macht. Der B-Mode wird überwiegend als erste Orientierung genutzt, um eine grobe Übersicht und einen ersten Eindruck der Herzfunktion zu bekommen. Er kann außerdem zur genauen Positionierung des Schallkopfes genutzt werden, um anschließend mit anderen Modi wie dem M-Mode oder der Doppler-Sonographie bestimmte Strukturen genauer zu untersuchen [32].

M-Mode: Der M-Mode liefert als eindimensionale Methode keine räumliche Darstellung des Herzens. Es wird ein schmaler Ultraschallstrahl ausgesendet, der die anatomischen Strukturen, die entlang dieser Linie liegen erfasst. Der Schallstrahl wird 
meist unter zweidimensionaler Kontrolle (B-Mode) platziert. Zur Standardisierung der Schnittachse in der PSLAX wird meist der Papillarmuskel als Orientierung für die Achse genutzt. Die Bewegung dieser anatomischen Strukturen wird mit Hilfe einer simultanen EKG-Aufzeichnung zeitlich zugeordnet. Diese Methode erreicht durch die Eindimensionalität im Vergleich zu den anderen Modi die höchste räumliche Auflösung. Sie eignet sich deshalb vor allem zur Beurteilung der statischen Dimensionen wie Wanddicke, Gefäß- und Ventrikeldurchmesser. Die Tiefe wird hierbei auf der vertikalen Achse abgebildet, während die Zeit auf der Abszissenachse dargestellt wird. Durch Nachzeichnen des Endokards und Epikards lassen sich auch Werte wie Ejektionsfraktion, Verkürzungsfraktion, Herzzeitvolumen und Schlagvolumen näherungsweise berechnen. Dennoch ist dieser Modus fehleranfällig, da die Volumenmessungen durch Berechnungen aus geometrischen Näherungsmodellen aus einem eindimensionalen Schnittbild über die Zeit erfolgt und nicht das gesamte Volumen abgebildet wird [33].

Doppler-Sonographie: Bei dieser Art der Sonographie wird mithilfe des Doppler-Effekts der Blutfluss in akustische Signale umgewandelt. Die Ultraschallwelle wird von den bewegten Objekten zum Beispiel den Erythrozyten je nach Flussgeschwindigkeit mit veränderter Frequenz reflektiert. Fließen die Erythrozyten auf den Schallkopf zu, so werden die ausgesendeten Ultraschallwellen mit höherer Frequenz reflektiert. Um die Flussrichtung deutlich zu machen, wird bei dieser Art der Sonographie die Fließrichtung farbkodiert. So werden die höherfrequenten Wellen, also das auf den Schallkopf zufließende Blut, rot dargestellt. Analog werden die niederfrequenten Wellen des abfließenden Blutes blau dargestellt. Die Kombination aus dem B-ModeBild und der Farbkodierung der Doppler-Sonographie wird als Duplex-Sonographie bezeichnet. Haupteinsatzgebiet der Duplex-Sonographie ist die Untersuchung der Gefäße auf Stenosen und Thrombosen sowie in der Echokardiographie die Beurteilung der Klappen zur Detektion von Vitien wie Stenosen oder Insuffizienzen. Turbulente Flüsse, durch Farbmosaike aus rot und blau zu erkennen, und eine erhöhte Flussgeschwindigkeit, durch die Farbe Grün gekennzeichnet, deuten hierbei auf Stenosen hin [34, 35].

EKV: EKV steht für EKG-synchronisierte Kilohertz Visualisierung. Hierfür werden die Bilder mehrerer Kontraktionszyklen gemittelt, sodass ein Cineloop mit deutlich 
verbesserter zeitlicher und räumlicher Auflösung produziert werden kann, als es im herkömmlichen B-Mode der Fall ist. Die zeitliche Auflösung von B-Mode-Bildern mit EKV kann eine Bildrate von bis zu $10.000 \mathrm{fps}$ (frames per second, Anzahl der Einzelbilder pro Sekunde) erreichen [19].

3D-Echokardiographie: Viele der Studien, die echokardiographische 2D-Daten mit Datensätzen aus MRTs vergleichen, kritisierten immer wieder die in der Sonografie fehlende Abbildung des gesamten Herzens [36, 37]. Hierdurch kann insbesondere bei pathologischer Herzfunktion selten eine exakte Messung gewährleistet werden und die Ergebnisse sind stets sehr stark vom Untersucher abhängig. Um echokardiographisch ähnlich exakte Messungen zu erzielen wie das CMR ist also ein Verfahren nötig, das wie im CMR auch in vielen Schnittachsen das gesamte Herz scannt und diese Bilder dann zu einem dreidimensionalen Bild zusammenfügen kann. Das Vevo2100®, der Vorläufer der für unsere Studie genutzten Imaging Plattform Vevo3100®, näherte sich einer Abbildung des gesamten Herzens an. Es handelt sich hierbei um ein Ultraschallgerät speziell für Kleintiere, das sowohl von der Hard- als auch von der Software auf 3D-Daten ausgelegt war. Hierfür wurde aus 2D Bildern von langer und kurzer Herzachse ein 3D-Bild rekonstruiert. In diesem Vevo2100®-System ist die Atemtriggerung (Respiratory Gating) und EKG-synchronisierte Sequenzierung bereits implementiert, sodass hiermit erstmals hochauflösende echokardiographische Bilder ohne atemabhängige Bewegungsartefakte erzeugt werden konnten. Hierdurch kann eine weitaus höhere Auflösung erreicht werden, als es mit den herkömmlichen 2D-Abbildungen der Herzaktion bis dahin möglich war.

Die Messungen aus 3D-Daten zeigten laut Ram et al. eine sehr gute Korrelation zu den Messungen aus CMR-Daten [14].

Einen ergänzenden Ansatz verfolgten Dawson et al.: Sie nutzten für ihre 3DMessungen eine Halterung für den Schallkopf, die manuell in $500 \mu \mathrm{m}$-Schritten entlang der kurzen Herzachse bewegt wurde. So wurde die kurze Achse an zwölf bis 18 verschiedenen Positionen gescannt. Die jeweilige Position des Schallkopfes wurde zur Auswertung herangezogen [38]. Diese Methode funktioniert ähnlich wie die des hier genutzten Vevo3100®, ist allerdings nicht standardisiert, da es sich um eine selbstgebaute Konstruktion ohne automatisierte Schnittbildgebung handelt. Die 
anschließende Auswertung der vielen bewegten Schnittbilder ist sehr aufwändig und fehleranfällig.

4D-Echokardiographie: Die seit Anfang 2016 verfügbare Vevo3100® Imaging Plattform baut auf dem 3D-System auf, es können nun 3D-Bilder über die Zeit, mit der Zeit als vierten Dimension, produziert werden. Es werden weiterhin atemabhängig getriggerte, EKG-synchronisierte Bilder erstellt. Neu ist aber, dass dieses System automatisch Schnittbilder mit einer Schrittweite von bis zu $50 \mu \mathrm{m}$ aufnehmen kann. Es ist in der Lage, automatisch einen vom Untersucher festgelegten Bereich in der gewählten Schichtdicke zu abzubilden. Umgesetzt wird dies durch einen Schrittmotor an dem der Schallkopf befestigt wird, und der in vorgegebenen Abständen Schnittbilder mittels EV aufnimmt. Somit kann man schrittweise Schnittbilder vom gesamten Herzen akquirieren. Mithilfe des aufgezeichneten EKGs, erfolgt im Anschluss eine räumliche und zeitliche Rekonstruktion zu einer 4D-Ansicht. Damit ist es erstmals möglich, den gesamten Ventrikel mit seinen Begrenzungen zu jedem Zeitpunkt des Herzzyklus sonographisch abzubilden und auszuwerten. Die Methode ähnelt der Methode des 4D CMRs, denn es werden automatisierte Schnittbilder über mehrere Herzzyklen aufgenommen und dann zu einem 3D-Bild des schlagenden Herzens zusammengefügt. 


\subsection{Kardiale Magnetresonanztomographie bei Nagetieren}

CMR gilt als der Goldstandard für kardiale Bildgebung an Nagetieren [39, 40]. Es ist durch automatisierte Schichtaufnahmen möglich, das Herz als 3D-Modell mit wenigen geometrischen Annahmen darzustellen [41].

Um Bewegungsartefakte zu minimieren, ist respiratorisches Gating und EKGSynchronisierung auch bei der CMR essenziell. Da CMR bereits einige Jahre an Nagetieren genutzt wird, sind die grundlegenden Prinzipien klar definiert und werden von den verschiedenen Forschungseinrichtungen befolgt:

(1) Die Tiere sind sediert, aber spontanatmend. Präferiertes Anästhetikum ist derzeit die Isofluran-Inhalation, da es nur minimale kardiodepressive Wirkung zeigt und schnell an- und abflutet [42].

(2) Die Herzfrequenz, Atemfrequenz und Körpertemperatur der Tiere werden überwacht und gegebenenfalls durch Anpassung der Anästhesie im physiologischen Bereich gehalten.

(3) Die Bilder werden mit respiratorischem Gating und EKG-Synchronisierung aufgenommen [39, 43].

(4) Die Positionierung der Nager muss reproduzierbar sein. Hierfür befinden sich die Tiere in Bauchlage. Mit Klebeband werden sie an einem Schlitten fixiert und bekommen über ein kegelförmiges Nasenstück das inhalative Anästhetikum verabreicht. Das EKG wird durch Elektroden an den Pfoten abgeleitet, der Atmungssensor ist meist am Abdomen fixiert. Die Temperatur wird in der Regel durch eine rektale Sonde überwacht [44].

Limitiert sind die Aufnahmen, wie beim Ultraschall auch, durch die geringe Körpergröße und die hohe Herzfrequenz der Tiere [45]. MRT-Systeme für Kleintiere unterscheiden sich durch eine höhere magnetische Flussdichte (Einheit: Tesla T) und Spulen mit kleinerem Innendurchmesser im Vergleich zu den üblicherweise für den Menschen genutzten MRTs. Eine höhere Flussdichte und die damit verbundenen höhere Feldstärke erhöht das Signal-Rausch-Verhältnis und führt zu einer höheren Auflösung, erhöht allerdings auch die Artefaktanfälligkeit [43].

Derzeit verfügbare Geräte ermöglichen eine zeitliche Auflösung von 10 bis 20 Phasen pro Herzzyklus sowie eine räumliche Auflösung von $0,1 \mathrm{~mm}$ bis $0,2 \mathrm{~mm}$. 


\section{Erläuterungen zur Publikation}

\subsection{Studienaufbau}

Ziel der Studie war der Vergleich der Daten aus der 4D-Echokardiographie mit dem Goldstandard 4D CMRs. Zusätzlich sollte eine Aussage über die Reproduzierbarkeit der 4DE-Daten getroffen werden, da insbesondere die 2DE-Messungen stark untersucherabhängig sind.

Um eine valide Aussage treffen zu können, wurden die Ratten in zwei Gruppen aufgeteilt:

(1) 20 Ratten wurden sowohl echokardiographischen Aufnahmen (2D und 4D) als auch 4D CMR unterzogen.

(2) Sechs weitere Ratten wurden zur Beurteilung der Reproduzierbarkeit der Echokardiographie eingesetzt. Diese sechs Ratten wurden hierzu von zwei unabhängigen Untersuchern sowie zweifach von demselben Untersucher geschallt. Insgesamt wurde die Studie an 26 jungen Sprague-Dawley Ratten aus eigener Zucht durchgeführt. Untersucht wurden die Ratten in einem Alter von vier Wochen, da diese Tiere so für weitere Experimente im Anschluss verwendet werden konnten. Es wurden gleichermaßen männliche und weibliche Tiere untersucht. Das Zeitintervall zwischen den Untersuchungen (4DE und 4D CMR) wurde mit $0 \pm 1$ Tag so gering wie möglich gehalten. Damit zusätzlich untersucht werden konnte, ob 4DE sensitiv genug ist, um geringe Abweichungen in der Herzfunktion zu detektieren, schlossen wir fünf herzkranke Tiere in unsere Studie ein. Um den kardialen Zufluss im Sinne eines Krankheitsmodells zu reduzieren, wurden hierfür fünf der 20 Ratten zwei Wochen vor den bildgebenden Aufnahmen einer Operation mit Ligatur der oberen und unteren Hohlvene unterzogen. Die 4D CMR wurde mit einem 7 T-Scanner durchgeführt, die 2D- und die 4D-Echokardiographie mit einem 40 MHz-Linearschallkopf. Beide Geräte sind speziell für die Untersuchung von Kleintieren entwickelt worden. Beide Verfahren wurden unter Inhalationssedierung mit 1,5\% Isofluran durchgeführt. Herz- und Atemfrequenz wurden die gesamte Zeit überwacht. Bei zu starken Abweichungen wurde durch Anpassung der Isoflurandosierung die Narkosetiefe reguliert. Eine rektal eingeführte Sonde maß dauerhaft die Körpertemperatur, sodass diese durch Regulierung des beheizten Tisches um $37,0 \pm 0,5^{\circ} \mathrm{C}$ stabil gehalten werden konnte. 


\subsection{Operation}

Als Krankheitsmodel für die Kontrollgruppe nutzen wir ein in unserer Arbeitsgruppe bereits etabliertes Modell der Herzinsuffizienz. Die Ratten werden hierfür im Alter von zwei Wochen einem operativen Eingriff unterzogen, in welchem die obere und untere Hohlvene herznah auf die Hälfte ihres Durchmessers reduziert wurden. Hierdurch wird der venöse Zufluss zum Herzen reduziert und damit eine Senkung des Herzzeitvolumens erreicht.

Die Ligatur der oberen und unteren Hohlvene wurde mithilfe eines Zeiss Mikroskops an $14 \pm 1$ Tage alten Ratten durchgeführt. 30 Minuten vor der Operation wurde Buprenorphin $(0,05-0,1 \mathrm{mg} / \mathrm{kg}$ Körpergewicht) intraperitoneal verabreicht. Die Narkose wurde durch $5 \%$ Isofluran eingeleitet. Die Ratten wurden unter Sicht mit einer 20-Gauche intravenösen Kanüle intubiert und anschließen mit einem PEEP (positiver endexpiratorischer Druck) von $10 \mathrm{~cm} \mathrm{H}_{2} \mathrm{O}$ und einer Atemfrequenz von $80-85$ pro Minute beatmet. Die Narkose wurde mit 1,5-2\% Isofluran fortgeführt.

Nach Entfernung der Thoraxbehaarung mit anschließender Desinfektion erfolgte ein Hautschnitt entlang des vierten Interkostalraums. Die Pleura wurde eröffnet und die Vena cava superior und inferior freipräpariert. Der Nervus phrenicus wurde geschont. Für die Ligatur wurde eine 16-Gauche intravenöse Kanüle mit einem äußeren Durchmesser von 0,6 mm genutzt, die als Platzhalter fungierte. Die Ligatur erfolgte unter Verwendung von Prolene-Nahtmaterial der Stärke 7-0. Daraufhin wurde der Thorax verschlossen und das Isofluran abgestellt. Sobald die Tiere suffizient und eigenständig atmeten, wurden sie extubiert. Bis sie vollständig wach und mobil waren, wurden sie unter einer Rotlichtlampe warmgehalten und nachfolgend wieder zum Muttertier gesetzt. In den 24 Stunden nach der Operation wurden die Tiere engmaschig beobachtet und es erfolgte eine Analgesie mit Buprenorphin.

\subsection{Ergebnisse}

Untersucht wurden die Korrelationen zwischen 4D CMR, 4DE und 2DE für die linksventrikuläre Ejektionsfraktion (LVEF), sowie die Reproduzierbarkeit der Echokardiographie. Die Ergebnisse lassen zwei Hauptaussagen treffen:

(1) Es konnte eine ausgezeichnete Korrelation zwischen den beide 4D Bildgebungen 4D CMR und 4DE gezeigt werden.

(2) 4DE ist kaum untersucherabhängig und dadurch sehr gut reproduzierbar. 
Aus (1) folgt, dass die 4DE Ergebnisse liefert, die genauso präzise wie die 4D CMR sind. Sowohl der Zeitaufwand, und damit die Sedierungsdauer für die Ratten, als auch der technische Aufwand sind bei der 4DE deutlich geringer. Die Ergebnisse zeigen, dass Forscher in Zukunft mit der Methode des 4DE eine gute Alternative zum 4D CMR haben.

\subsection{Ausblick}

Die Möglichkeit, das gesamte Herz mittels Ultraschall räumlich aufgelöst abzubilden, ist ein großer Fortschritt. Der bisher größte Nachteil der Echokardiographie, durch geometrische Annäherungen große Abweichungen der Messungen zu induzieren, wird somit weitestgehend vermieden. Von der verkürzten Untersuchungszeit dieser Methode profitieren vor allem longitudinale Studien, bei denen mehrfache Untersuchungen notwendig sind.

Die Vevo3100® Imaging Plattform wurde speziell für Kleintiere entwickelt. Die Übertragbarkeit dieser Ergebnisse auf Untersuchungen am Menschen ist daher nicht direkt gegeben. Die hohen Frequenzen, die diese hohe Auflösung ermöglichen, erreichen beim Menschen keine ausreichende Eindringtiefe.

Dennoch ist es denkbar, das technische Prinzip der Methode auf die Diagnostik bei Menschen zu übertragen. 


\section{Publikation}

Stegmann H, Bäuerle T, Kienle K, Dittrich S, Alkassar M. 4D cardiac magnetic resonance imaging, 4D and 2D transthoracic echocardiography: a comparison of in-vivo assessment of ventricular function in rats. Lab Anim. $2019 \mathrm{Apr} ; 53(2): 169-179$. doi:

10.1177/0023677218789971. Epub 2018 Aug 6. PMID: 30081741.

https://pubmed.ncbi.nlm.nih.gov/30081741/ 


\section{Referenzen}

1. Tsai, L.L., et al., A Practical Guide to MR Imaging Safety: What Radiologists Need to Know. Radiographics, 2015. 35(6): p. 1722-37.

2. Greupner, J., et al., Head-to-head comparison of left ventricular function assessment with 64-row computed tomography, biplane left cineventriculography, and both 2- and 3-dimensional transthoracic echocardiography: comparison with magnetic resonance imaging as the reference standard. J Am Coll Cardiol, 2012. 59(21): p. 1897-907.

3. Martinez, G.V., Introduction to MRI Physics. Methods Mol Biol, 2018. 1718: p. 3-19.

4. Plewes, D.B. and W. Kucharczyk, Physics of MRI: a primer. J Magn Reson Imaging, 2012. 35(5): p. 1038-54.

5. Gutberlet, M., et al., Comparison of different cardiac MRI sequences at 1.5 T/3.0 Twith respect to signal-to-noise and contrast-to-noise ratios - initial experience. Rofo, 2004. 176(6): p. 801-8.

6. Fathi, A., et al., Effects of contrast administration on cardiac MRI volumetric, flow and pulse wave velocity quantification using manual and software-based analysis. $\mathrm{Br} \mathrm{J}$ Radiol, 2018. 91(1084): p. 20170717.

7. Kanal, E., et al., Standardized MR terminology and reporting of implants and devices as recommended by the American College of Radiology Subcommittee on MR Safety. Radiology, 2015. 274(3): p. 866-70.

8. Nospes, S., W. Mann, and A. Keilmann, [Magnetic resonance imaging in patients with magnetic hearing implants: overview and procedural management]. Radiologe, 2013. 53(11): p. 1026-32.

9. McGuirt, D., Alternatives to Sedation and General Anesthesia in Pediatric Magnetic Resonance Imaging: A Literature Review. Radiol Technol, 2016. 88(1): p. 18-26.

10. Mohamed, A.A., A.A. Arifi, and A. Omran, The basics of echocardiography. J Saudi Heart Assoc, 2010. 22(2): p. 71-6.

11. Shriki, J., Ultrasound physics. Crit Care Clin, 2014. 30(1): p. 1-24, v.

12. Lecluyse, V., Understanding the physics and fluid dynamics governing ultrasound gel behaviour facilitates its use: the ketchup analogy! Can J Anaesth, 2015. 62(4): p. 4278.

13. Le, H.T., et al., Imaging Artifacts in Echocardiography. Anesth Analg, 2016. 122(3): p. 633-46.

14. Ram, R., et al., New approaches in small animal echocardiography: imaging the sounds of silence. Am J Physiol Heart Circ Physiol, 2011. 301(5): p. H1765-80.

15. Garg, R., et al., Observer variation in the echocardiographic measurement of maximum atrial septal excursion: a comparison of M-mode with two-dimensional or transesophageal echocardiography. Echocardiography, 2009. 26(10): p. 1122-6.

16. Morbach, C., et al., Impact of acquisition and interpretation on total inter-observer variability in echocardiography: results from the quality assurance program of the STAAB cohort study. Int J Cardiovasc Imaging, 2018. 34(7): p. 1057-1065.

17. Pepi, M., et al., Head-to-head comparison of two- and three-dimensional transthoracic and transesophageal echocardiography in the localization of mitral valve prolapse. $\mathrm{J}$ Am Coll Cardiol, 2006. 48(12): p. 2524-30.

18. Khanna, D., et al., Transthoracic and transesophageal echocardiographic assessment of mitral regurgitation severity: usefulness of qualitative and semiquantitative techniques. Echocardiography, 2005. 22(9): p. 748-69.

19. Wang, L.W., et al., High-Frequency Echocardiography- Transformative Clinical and Research Applications in Humans, Mice, and Zebrafish. Circ J, 2018. 82(3): p. 620628. 
20. Critical care ultrasound: coming soon to an ICU near you. J Am Soc Echocardiogr, 2012. 25(1): p. 25A.

21. Hasenfuss, G., Animal models of human cardiovascular disease, heart failure and hypertrophy. Cardiovasc Res, 1998. 39(1): p. 60-76.

22. Russell, J.C. and S.D. Proctor, Small animal models of cardiovascular disease: tools for the study of the roles of metabolic syndrome, dyslipidemia, and atherosclerosis. Cardiovasc Pathol, 2006. 15(6): p. 318-30.

23. Camacho, P., et al., Small mammalian animal models of heart disease. Am J Cardiovasc Dis, 2016. 6(3): p. 70-80.

24. Shimoyama, M., et al., Rat Genome and Model Resources. ILAR J, 2017. 58(1): p. 4258.

25. Worthey, E.A., A.J. Stoddard, and H.J. Jacob, Sequencing of the rat genome and databases. Methods Mol Biol, 2010. 597: p. 33-53.

26. Patten, R.D. and M.R. Hall-Porter, Small animal models of heart failure: development of novel therapies, past and present. Circ Heart Fail, 2009. 2(2): p. 138-44.

27. Aitman, T.J., et al., Progress and prospects in rat genetics: a community view. Nat Genet, 2008. 40(5): p. 516-22.

28. Coatney, R.W., Ultrasound imaging: principles and applications in rodent research. ILAR J, 2001. 42(3): p. 233-47.

29. Lang, R.M., et al., Recommendations for cardiac chamber quantification by echocardiography in adults: an update from the American Society of Echocardiography and the European Association of Cardiovascular Imaging. Eur Heart J Cardiovasc Imaging, 2015. 16(3): p. 233-70.

30. Wandt, B., et al., Echocardiographic assessment of ejection fraction in left ventricular hypertrophy. Heart, 1999. 82(2): p. 192-8.

31. Arias, T., et al., Comparison of echocardiographic measurements of left ventricular volumes to full volume magnetic resonance imaging in normal and diseased rats. J Am Soc Echocardiogr, 2013. 26(8): p. 910-8.

32. Thijssen, J.M. and C.L. de Korte, Cardiological Ultrasound Imaging. Curr Pharm Des, 2014. 20(39): p. 6150-61.

33. Feigenbaum, H., Role of M-mode technique in today's echocardiography. J Am Soc Echocardiogr, 2010. 23(3): p. 240-57; 335-7.

34. Gardin, J.M., Pulsed Doppler Echocardiography: An Historical Perspective. J Am Soc Echocardiogr, 2018. 31(12): p. 1330-1343.

35. Baker, D.W., S.A. Rubenstein, and G.S. Lorch, Pulsed Doppler echocardiography: principles and applications. Am J Med, 1977. 63(1): p. 69-80.

36. Kaminska, H. and B. Werner, Three-dimensional echocardiography in the assessment of ventricular function in children: pros, cons, and hopes. Kardiol Pol, 2019. 77(1): p. 12-17.

37. Bertrand, P.B., et al., Fact or Artifact in Two-Dimensional Echocardiography: Avoiding Misdiagnosis and Missed Diagnosis. J Am Soc Echocardiogr, 2016. 29(5): p. 381-91.

38. Dawson, D., et al., Quantitative 3-dimensional echocardiography for accurate and rapid cardiac phenotype characterization in mice. Circulation, 2004. 110(12): p. 16327.

39. Richardson, J.D., et al., Cardiac magnetic resonance, transthoracic and transoesophageal echocardiography: a comparison of in vivo assessment of ventricular function in rats. Lab Anim, 2013. 47(4): p. 291-300.

40. Prunier, F., et al., Serial magnetic resonance imaging based assessment of the early effects of an ACE inhibitor on postinfarction left ventricular remodeling in rats. Can $\mathrm{J}$ Physiol Pharmacol, 2005. 83(12): p. 1109-15. 
41. Schneider, J.E., et al., Fast, high-resolution in vivo cine magnetic resonance imaging in normal and failing mouse hearts on a vertical 11.7 T system. J Magn Reson Imaging, 2003. 18(6): p. 691-701.

42. Hanusch, C., S. Hoeger, and G.C. Beck, Anaesthesia of small rodents during magnetic resonance imaging. Methods, 2007. 43(1): p. 68-78.

43. Kramer, M., et al., Self-gated cardiac Cine MRI of the rat on a clinical 3 T MRI system. NMR Biomed, 2015. 28(2): p. 162-7.

44. Bunck, A.C., et al., Feasibility of functional cardiac MR imaging in mice using a clinical 3 Tesla whole body scanner. Invest Radiol, 2009. 44(12): p. 749-56.

45. Jeuthe, S., et al., Assessment of cardiac function and myocardial morphology using small animal Look-Locker inversion recovery (SALLI) MRI in rats. J Vis Exp, 2013(77). 


\section{Abkürzungsverzeichnis}

EDV Enddiastolisches Volumen

EKV EKG-synchronisierte Kilohertz Visualisierung

ESV Endsystolisches Volumen

IVC Vena cava inferior

LV Linker Ventrikel

LVEF Linksventrikuläre Ejektionsfraktion

MRT Magnetresonanztomographie

PSAX Parasternale kurze Achse

PSLAX Parasternale lange Achse

SVC Vena cava superior

TTE Transthorakale Echokardiographie

4D CMR Vierdimensionale kardiale Magnetresonanztomographie

2DE Zweidimensionale Echokardiographie

3DE Dreidimensionale Echokardiographie

4DE Vierdimensionale Echokardiographie 


\section{Danksagung}

Die Forschungsarbeit, die zu der vorliegenden Dissertation führte, wurde zwischen Februar 2016 und September 2020 in der Kinderkardiologie des Uniklinikums Erlangen unter der Leitung von Prof. Dr. Sven Dittrich durchgeführt.

Mein herzlicher Dank gilt an erster Stelle meinem Doktorvater Prof. Dr. Sven Dittrich für die Überlassung des Promotionsthemas und die Möglichkeit, in diesem äußerst spannenden Gebiet zu forschen. Danke auch für die vielen lobenden Worte und die stets sehr konstruktive Kritik, die zur Fertigstellung dieser Promotion geführt haben. Meinem Arbeitsgruppenleiter und Betreuer Dr. Muhannad Alkassar möchte ich danken für das entgegengebrachte Vertrauen und die Unterstützung bei allen Schritten dieses Weges, von den Beginnen im Tierstall, über die Auswertung der Daten bis zum Verfassen der Publikationsschrift. Ausdrücklich bedanken möchte ich mich zudem bei Katharina Kienle, die mir sowohl während der Arbeit im Tierstall durch das Operieren der Tiere als auch bei der Auswertung eine verlässliche Hilfe war und mir über die Jahre zu einer guten Freundin geworden ist.

Besonders bedanken möchte ich mich außerdem bei Manuel Böhm und meiner Familie für die Unterstützung und Wertschätzung meiner Arbeit an der Dissertation.

Danken möchte ich zum Schluss noch meinem kanadischen Gastvater Evan Thornton, der stets bereit war, die englischen Texte Korrektur zu lesen und eine sehr zuverlässige Hilfe für mich war. 